\title{
Novel Features of dFMR 1, the Drosophila Orthologue of the Fragile X Mental Retardation Protein
}

\author{
Annette Schenck, Veronique Van de Bor, Barbara Bardoni, \\ and Angela Giangrande ${ }^{1}$ \\ Institut de Génétique et de Biologie Moléculaire et Cellulaire, CNRS/INSERM/ULP, \\ B.P.163, 67404 Illkirch Cedex, C.U. de Strasbourg, France
}

Received October 1, 2001; revised April 22, 2002; accepted for publication April 30, 2002

\begin{abstract}
FMRP belongs to a family of widely expressed proteins that contain RNA-binding domains. Although lack of human FMRP results in mental retardation, correlated with subtle synaptic changes, the precise role of FMRP remains elusive. The Drosophila genome contains a single gene homologous to the FXR family. We show that dFMR 1 is subjected to transcriptional and posttranscriptional regulation during development and that it homomerizes, like its human counterpart. dFMR 1 profile of expression recapitulates that of the human FXR protein family: it is highly enriched in muscles, in central nervous system and in gonads. In the larval brain, anti-dFMR 1 also recognizes mushroom bodies, a centre that mediates learning and memory. These features make the fly an ideal system to analyse the role of the FXR family and to identify genes in the FMRP pathway. $\odot 2002$ Elsevier Science (USA)
\end{abstract}

Key Words: fragile $X$ syndrome; mental retardation; FMRP; Drosophila melanogaster; dFMR 1; posttranslational modification.

\section{INTRODUCTION}

Despite extensive research in the last decade, the mechanisms by which the absence of the fragile $X$ Mental Retardation Protein (FMRP) result in the human disorder Fragile $X$ Syndrome are still elusive. Fragile $X$ Syndrome affects 1 in 4000 males and 1 in 7000 females and is characterised by mental retardation, abnormal behavioural manifestations and enlarged testis (for review see (Bardoni et al., 2000)). At the neuronal level, patients exhibit abnormal shape and number of dendritic spines (Irwin et al., 2000), a feature also observed in the FMR1 knockout mouse model (Comery et al., 1997), suggesting that abnormal maturation of neuronal connectivity may underlie the mental impairment. FMRP is an RNA-binding protein containing two KH-domains and a RGG box (Ashley et al., 1993; Siomi et al., 1993), both typical RNA-binding motifs. Indeed, FMRP has been recently shown to

\footnotetext{
${ }^{1}$ To whom correspondence and reprint requests should be addressed. Fax: (33)388653201. E-mail: angela@titus.u-strasbg.fr.
}

bind a purine quartet motif in its own mRNA (Schaeffer et al., 2001) with high affinity. Although it is widely expressed, FMRP is particularly abundant in neurons in various regions of the brain, including hippocampus, whose importance in learning and memory is well known. Two FMRP related proteins, termed FXR1P and FXR2P, exist in humans (Siomi et al., 1996; Zhang et al., 1995). These two proteins, which show high similarity with FMRP, are endowed with the same functional motifs and can interact with themselves or with FMRP to form homo- or heteromers. Currently, it is not yet clear whether the three proteins (FXR proteins) have distinct or overlapping functions. Evidence that FMRP can act as a translational regulator (Laggerbauer et al., 2001; Li et al., 2001; Brown et al., 2001; Darnell et al., 2001) notably at the level of neuronal synapses (Weiler et al., 1997; Greenough et al., 2001) is currently accumulating. However, downstream (mRNA) targets (Ashley et al., 1993; Sung et al., 2000; Schaeffer et al., 2001; Brown et al., 2001; Darnell et al., 2001) as well as candidates for proteins that may participate in an regulation of FMRP function 
(Schenck et al., 2001) are just beginning to emerge. A single Drosophila melanogaster orthologue of the human FXR family, similarly related to FMRP, FXR1P, and FXR2P, has been recently identified (Wan et al., 2000). Genetic tools applicable to Drosophila make this organism a powerful model to determine the precise function of this protein family, and to identify genetic modifiers and thus pathways, in which FMRP-like proteins are involved.

Here, we present novel data on the Drosophila orthologue, dFMR1 of the FXR family. We describe a dFMR1 splice variant and present data strongly suggesting the existence of a posttranslational modification of dFMR1 dynamically regulated during embryonic development. We have analysed the distribution of dFMR1 protein and transcript at the subcellular level and also during development. We show that anti-dFMR1 also recognizes Drosophila mushroom bodies, a brain structure conserved throughout the insect kingdom that mediates learning and memory in lower organisms.

The results presented here reinforce the hypothesis that the molecules expressed and required for the organization of nervous system are conserved among vertebrates and invertebrates.

\section{MATERIALS AND METHODS}

Fly stocks. All experiments were performed on embryos or larvae of the wild-type strain Sevelen.

Protein extracts. S2 cells were cultured in Schneider cell medium (Gibco BRL) $+10 \%$ fetal calf serum. Cells were scraped in this medium and washed three times with $1 \times$ PBS. The pellet was lysed in $1 \times$ SDS-PAGE loading buffer and subjected to SDS-PAGE analysis. For embryonic extracts, staged embryos were mashed with a pestle in buffer A $(150 \mathrm{mM} \mathrm{NaCl}, 20 \mathrm{mM}$ Tris $\cdot \mathrm{HCl}$, $\mathrm{pH}$ 7.5, $1 \mathrm{mM}$ EDTA, 0, $1 \mathrm{mM} \mathrm{MgCl}_{2}, 1 \%$ Triton $\mathrm{X}-100$, protease inhibitor cocktail). Stages are indicated in hours after egg laying (ael) at $25^{\circ} \mathrm{C}$. The supernatant of a $12,000 \mathrm{~g}$ centrifugation was briefly sonicated, and the amount of total protein was determined by Bradford assay.

Antibody production and purification. For the production of dFMR1 polyclonal antibodies (pAb) two synthetic polypeptides EELREEAQKDGIHKEFQRTI (position 137-156, pAb 1718) and RNKNRSNNHTDQPSGQQ (position 649-665, pAb 1717) were used. Both peptides have an additional cysteine in the Cterminus. The peptides were linked to ovalbumine (ovalbumine-MBS; Aldrich) via this cysteine residue and subsequently purified from the free polypeptide. Rabbits were immunised by intradermal injections of $100 \mu \mathrm{g}$ of coupled peptide. Antibodies were purified on affinity columns (Sulfolink Coupling gel) coupled to the same peptides used for immunisation, according to manufacturer's instructions (Pierce). Antibodies were eluted from the column, collected in fractions and tested separately on S2 cell extract by western blot analysis. Fractions of pAb 1717 that exclusively detected a $85 / 92-\mathrm{kDa}$ doublet were pooled, subjected to dialysis against $1 \times$ PBS, and are referred to as antidFMR1.

SDS-PAGE analysis. Proteins were separated in $10 \%$ Polyacrylamide gels. Gel electrophoresis, Coomassie staining, and Western blotting (WB) were performed according to standard procedures. pAbs 1717 and 1718 were used in WB analysis at 1:200 and 1:4000, respectively.

Mass spectrometry analysis. MALDI-TOF mass spectrometry analysis was carried out as described in (Gadroy et al., 1998).

GST-pull down assay. The dFMR1 coding sequence was amplified by PCR using the primers GGGGAATTCGAAGATCTCCTCGTGGAAGTTC, GGGTTGGCGGCCGCTTAGGACGTGCCATTGACCAG and cloned in frame with the N-terminal Glutathione $S$ transferase tag in the vector pGEX-4T-1 as an EcoRI/NotI fragment. Overexpression of GST-dFMR1 or GST alone (using the empty pGex vector) was induced by $1 \mathrm{mM}$ IPTG for $3.5 \mathrm{~h}$ at room temperature. Purification was carried out as described in (De Santa Barbara et al., 1998). The pull-down assay was performed in the presence of $150 \mathrm{mM} \mathrm{NaCl}$ as in Bardoni et al. (1999) using approximately $3 \mu \mathrm{g}$ of GST-dFMR1 fusion protein or GST alone.

Immunolabeling and in situ hybridization. Fixation, dissection and antibody incubation were performed as in (Vincent et al., 1996). In situ hybridization were performed as in (Bernardoni et al., 1997) using a fulllength dFMR1 specific riboprobe. A dFMR1 exon 12 specific probe revealed the same pattern, with a lower intensity. Embryos and dissected larvae were mounted in Vectashield medium (Vector laboratories). The following primary antibodies were used: $\mathrm{pAb}$ 1717 (1:40), anti-Elav (1:100) (provided by G. Rubin), and 22C10 (1:20) (provided by S. Benzer). Secondary antibodies coupled with Cy3 or FITC (Jackson) were used at 1:400.

Microscopy. The light microscope was a Zeiss Axiophot2. Confocal images were obtained using a Leica TCS4D microscope. Images were assembled using an in-house developed software, TCSTK/TIMT. 


\section{RESULTS}

\section{Distinct Forms of dFMR 1}

To study the expression of dFMR1, the single Drosophila orthologue representing the FXR family in Drosophila, we raised antibodies against two synthetic peptides corresponding to amino acids (aa) 137-156 and 649-665 in dFMR1 (see material and methods). Sequence searches through databases indicated no homology to any other sequence in the fly genome. In Western blot analysis of Drosophila S2 cell extract, both antibodies showed the same pattern of a doublet of approximately 85 and $92 \mathrm{kDa}$. Figure 1A shows a representative western blot probed with pAb 1717. Previously, only one band of around $85 \mathrm{kDa}$ was described (Wan et al., 2000), which we believe corresponds to our $85-\mathrm{kDa}$ signal. A possible explanation for the presence of a doublet in western blot is the occurrence of alternative splicing (human FMR1 gene also undergoes extensive alternative splicing), even though this has not been detected for dFMR1 (Wan et al., 2000). By analyzing cDNA sequences available in the databases (NCBI Gene Bank, Acc. No. AF305881 and AJ271221), we did find that two different acceptor sites of exon 9 at the exon $8 / 9$ junction can be used, generating proteins that differ for the presence of three amino acids (VSA) within the KH2 domain at position 321 of the dFMR1 protein. We also sequenced two EST clones, one of them representing the version without $\left(\mathrm{dFMR} 1_{-\mathrm{VSA}}\right)$, the other the version, including the three aa $\left(\mathrm{dFMR} 1_{+\mathrm{VSA}}\right)$.

Polyclonal antibody 1717 efficiently immunoprecipitates the two recognized proteins, which enabled us to assess whether presence/absence of three amino acids acounts for the 85- and 92-kDa bands and to exclude any possibility of antibody-crossreactivity. Herefore, we performed large scale immunoprecipitation from S2 cell extract and separated the 85/92-kDa bands by SDS-PA gel electrophoresis. The Coomassie blue-stained bands were treated with trypsin and separately subjected to mass spectrometry (ms) analysis. Figures 1B and 1D list the dFMR1 peptides derived from the 85- and 92-kDa proteins. This analysis revealed that indeed both bands represent the dFMR1 protein. It also showed that both mRNA splice variants are translated into protein and exist in significant amounts. However, none of the two splice variants is responsible for the 92-kDa band in Western blot analysis since peptides corresponding to dFMR $1_{-\mathrm{VSA}}$ and $\mathrm{dFMR} 1_{+\mathrm{VSA}}$ peptides have been both identified by analysis of the $85 \mathrm{kD}$ material (Fig. 1B, peptide sequences underlined).

The identified peptides derived from tryptic digest of the $85-\mathrm{kDa}$ band covered more than $62 \%$ of the dFMR1 protein and include sequences from 9 of the 10 coding exons (exons 4-12) (Fig. 1C). Identification of a peptide corresponding to positions 18-47 further excludes the possibility that in the $85-\mathrm{kDa}$ product translation starts from a down stream methionine (as for example methionine 39 or 65).

Finally, WB analysis of Drosophila S2 cells transiently overexpressing dFMR1 $1_{-\mathrm{VSA}}$ showed increased signal at 85- and 92-kDa bands (Fig. 1E), clearly demonstrating that both protein forms can be produced from a single coding sequence.

For these reasons, and in agreement with findings by Wan et al. (2000), the $85-\mathrm{kDa}$ band must already represent a full-length dFMR1 protein (dFMR1 $1_{-\mathrm{VSA}}$ and $\left.\mathrm{dFMR} 1_{+\mathrm{VSA}}\right)$, strongly suggesting that the $92-\mathrm{kDa}$ band results from posttranslational modifications of full-length dFMR1. We then speculated that the 92$\mathrm{kDa}$ band represented a phosphorylated form of dFMR1. In a dephosphorylation experiment, however, treatment of immunopurified dFMR1 with either acid or alkaline phosphatase did not lead to a shift of intensity between the 85- and 92-kDa band (data not shown), favoring the idea of posttranslational modifications different from phosphorylation. Also, ms analysis of the $92-\mathrm{kDa}$ band gave no indication for the presence of phosphorylated dFMR1 peptides.

Remarkably, an expression analysis throughout embryonic development (Fig. 2A) shows that the 92-kDa dFMR1 is almost exclusively present in a discrete period during embryogenesis (9-12 h ael), suggesting dynamic regulation and functional relevance of this second, modified dFMR1 form.

\section{dFMR 1 Homomerization}

In humans, FMRP, FXR1P, and FXR2P form heteroand homomers via an interaction site localized in the $\mathrm{N}$-terminus of the proteins (Siomi et al., 1996). Recently, it was reported that the only known point mutation (1304N, localized within the KH2 domain) in the human FMRP protein in a patient severely affected by Fragile X Syndrome abolishes its homomerization (Laggerbauer et al., 2001). Therefore, the ability to homomerise must contribute in an important way to FMRP function.

To address the question as to whether the dFMR $1_{-\mathrm{VSA}}$ and dFMR $1_{+\mathrm{VSA}}$ splice variants exhibit different homomerisation properties, we performed a 




B

$85 \mathrm{kD}$ :

\begin{tabular}{|c|c|c|c|c|}
\hline \multicolumn{2}{|c|}{ Region } & \multirow{2}{*}{$\begin{array}{l}\text { Predicted } \\
3240.6\end{array}$} & \multirow{2}{*}{$\begin{array}{l}\text { Observed } \\
3240.7\end{array}$} & \multirow{2}{*}{$\begin{array}{l}\text { Peptide Sequence (and adjacent residues) } \\
\text { (K) GQVTAVADDGIFVDVDGVPESMKYPFVNVR (L) }\end{array}$} \\
\hline 18 & -47 & & & \\
\hline 48 & -72 & 2818.4 & 2818.5 & (R) LPPEETVEVAAPIFEEGMEVEVFTR ( $T$ ) \\
\hline 77 & -87 & 1362.7 & 1362.6 & (R) ETCGWWVGIIK (M) \\
\hline 127 & -145 & 2325.1 & 2325.1 & (K) TPYQFTLPVPEELREEAQK (D) \\
\hline 146 & -154 & 1129.6 & 1129.6 & (K) DGIHKEFQR (T) \\
\hline 155 & -165 & 1269.6 & 1269.6 & (R) TIDAGVCNYSR (D) \\
\hline 176 & -182 & 945.5 & 945.5 & (K) FEHTQRR (A) \\
\hline 183 & -192 & 1267.6 & 1267.6 & (R) ASMLKDMHFR (N) \\
\hline 220 & -227 & 1013.5 & 1013.5 & (R) GNYVEBFR (V) \\
\hline 228 & -248 & 2181.1 & 2181.2 & (R) VRDDLMGLAIGSHGSNIQAAR (T) \\
\hline 249 & -261 & 1446.7 & 1446.7 & (R) TVDGVTNIELEEK (S) \\
\hline 267 & -277 & 1234.6 & 1234.6 & (K) ISGETEESVQR (A) \\
\hline 280 & -293 & 1729.8 & 1729.9 & (R) AMLEYAEEFFQVPR (E) \\
\hline 306 & -313 & 957.6 & 957.6 & (R) IIQEIVDK (S) \\
\hline 321 & -336 & 1727.8 & 1727.7 & (K) VSAIAGDDEQDQNIPR (E) \\
\hline 324 & -336 & 1470.7 & 1470.7 & (K) IAGDDEQDQNIPR ( $\mathrm{B})$ \\
\hline 337 & -356 & 2142.2 & 2142.2 & (R) BLAHVPFVFIGTVESIANAK (V) \\
\hline 357 & -367 & 1351.8 & 1351.7 & (K) VLLEYHLSHLK (B) \\
\hline 368 & -376 & 1158.6 & 1158.6 & (K) EVEQLRQEK (M) \\
\hline 377 & -384 & 1032.5 & 1032.5 & (K) MEIDQQLR (A) \\
\hline 385 & -401 & 1825.9 & 1825.9 & (R) AIQESSMGSTQSFPVTR (R) \\
\hline 406 & -415 & 1112.5 & 1112.5 & (R) GYSSDIESVR (S) \\
\hline 446 & -451 & 589.4 & 859.4 & (R) YHNNRR (D) \\
\hline 451 & -459 & 1169.5 & 1169.4 & (R) RDEDDYNSR (G) \\
\hline 514 & -526 & 1665.7 & 1665.7 & (R) DYQHHNHTTEEVR (E) \\
\hline 537 & -548 & 1259.5 & 1259.5 & (R) ADSNSSYEGSSR (R) \\
\hline 555 & -590 & 3723.8 & 3723.8 & (K) NNNGPSNTNGAVANNNNKPQSAQQPQQQQPPAPGN \\
\hline 601 & -616 & 1471.7 & 1471.7 & (K) QNSGNANAAGGASKPK (D) \\
\hline 621 & -651 & 3486.7 & 3486.7 & (R) NGDKQQAGTQQQQPSQVQQQQAAQQQQPKPR (R) \\
\hline 657 & -676 & 2223.1 & 2223.1 & (R) SNNHTDQPSGQQQLAENVKK (E) \\
\hline
\end{tabular}

D

\section{$92 \mathrm{kD}$ :}

\begin{tabular}{|c|c|c|c|c|}
\hline \multicolumn{2}{|c|}{ Region } & \multirow{2}{*}{$\begin{array}{c}\text { Predicted } \\
1362.7\end{array}$} & \multirow{2}{*}{$\begin{array}{c}\text { Observed } \\
1362.6\end{array}$} & \multirow{2}{*}{$\begin{array}{l}\text { Peptide Sequence (and adjacent residues) } \\
\text { (R) ETCGWWVIIK (M) }\end{array}$} \\
\hline 77 & -87 & & & \\
\hline 146 & -154 & 1129.6 & 1129.6 & (K) DGIHKEFQR (T) \\
\hline 155 & -165 & 1269.6 & 1269.6 & (R) TIDAGVCNYSR (D) \\
\hline 176 & -182 & 945.5 & 945.5 & (K) FBHTQKR (A) \\
\hline 183 & -192 & 1267.6 & 1267.6 & (R) ASMLKDMHFR (N) \\
\hline 267 & -277 & 1234.6 & 1234.6 & (K) ISGETEESVQR (A) \\
\hline 280 & -293 & 1729.8 & 1729.9 & (R) AMLEYAEEFFQVPR (E) \\
\hline 306 & -318 & 1503.9 & 1503.8 & (R) IIQEIVDKSGVFR (I) \\
\hline 319 & -336 & 1711.9 & 1711.8 & (R) IKIAGDDEQDQNIPR (E) \\
\hline 385 & -401 & 1825.9 & 1825.9 & (R) AIQESSMGSTQSFPVTR (R) \\
\hline 469 & -483 & 1588.7 & 1588.7 & (R) GYNDRGGGDNTGSYR (G) \\
\hline 514 & -526 & 1665.7 & 1665.7 & (R) DYQHHNHTTEEVR (E) \\
\hline 621 & -651 & 3486.7 & 3486.7 & (R) NGDKQQAGTQQQQPSQVQQ \\
\hline 657 & -676 & 2223.1 & 2223.1 & (R) SNNHTDQPSGQQC \\
\hline
\end{tabular}

FIG. 1. Distinct dFMR1 forms in Drosophila. (A) Western blot analysis of Drosophila S2 cell line extract using pAb \#1717. Two bands of 85 and 92 $\mathrm{kDa}$ were detected. (B, D) Results of mass spectrometry analysis of the 85- and 92-kD bands after immunopurification by pAb \#1717, respectively. (B) Note the two underlined peptides, the presence of which demonstrates that both splice variants of dFMR1 (dFMR1 $1_{-\mathrm{VSA}}$ and $\left.\mathrm{dFMR} 1_{+\mathrm{VSA}}\right)$ can appear with an apparent molecular weight of $85 \mathrm{kDa}$. (C) dFMR1 full-length sequence (dFMR $\left.1_{+\mathrm{VSA}} / \mathrm{dFMR} 1_{-\mathrm{vSA}}\right)$. All peptides identified by analysis of the $85-\mathrm{kDa}$ material (listed in B) are underlined. Exon junctions are indicated by double-headed arrows and numbers of the corresponding exons. At the exon 8/9 junction, absence of the three amino acids VSA results in the dFMR1 $1_{-v S A}$ splice variant. (E) S2 cell extracts from nontransfected cells (left) and from cells that are transiently transfected with a dFMR1 $1_{-\mathrm{VSA}} / \mathrm{pPAc}$ construct (right). Total amount of protein loaded per lane: $140 \mu \mathrm{g}$ (left) and $40 \mu \mathrm{g}$ (right). Note that, in transfected cells both bands are present at higher levels than in non transfected cells.

GST-pull down experiment (Fig. 2B) using recombinant GST-tagged dFMR1 $1_{-\mathrm{VSA}}$ and $\mathrm{dFMR} 1_{+\mathrm{VSA}}$ proteins and their in vitro translated nontagged counterparts, human FMRP and luciferase as positive (Wan et al., 2000) and negative control, respectively. Binding assays were performed in the presence of $150 \mathrm{mM} \mathrm{NaCl}$, 
A

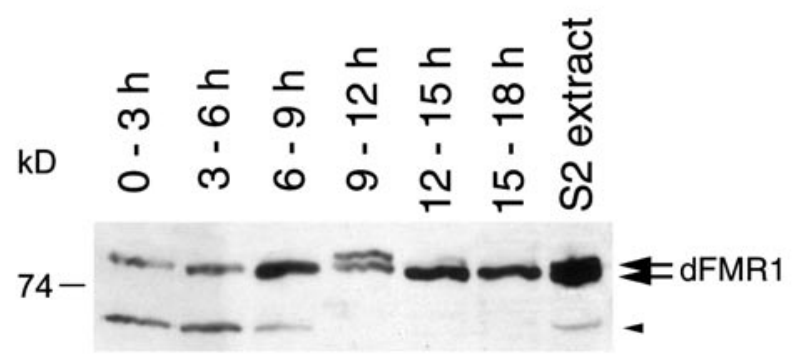

B

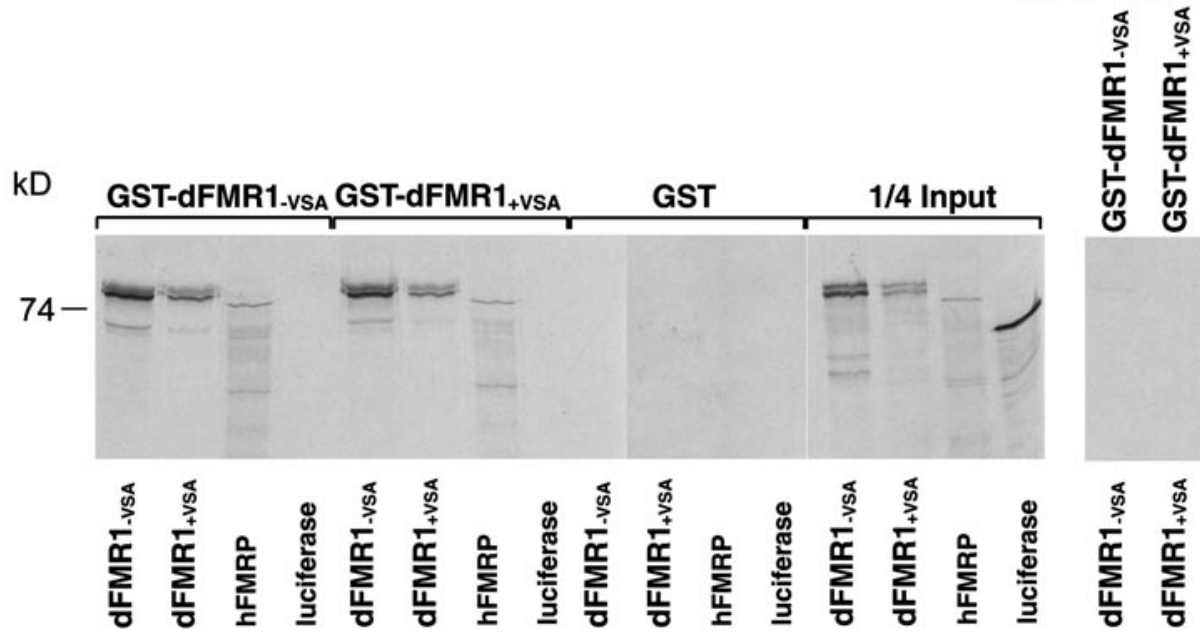

FIG. 2. Developmental profile and homomerisation properties of dFMR1. (A) dFMR1 expression during embryonic development. Extracts of staged embryos were analysed for dFMR1 expression using western blotting with pAb \#1717. Equivalent amounts of protein extracts were loaded. Embryonic stages are indicated at top as hours after egg laying (ael). The 85-kDa band was detectable throughout embryonic development. In contrast, the $92-\mathrm{kDa}$ band is almost exclusively restricted to $9-12 \mathrm{~h}$ embryos. A third product (arrowhead) of approximately $65 \mathrm{kDa}$ is detected at early stages, the identity of which was not further investigated. (B) The FMR1 protein is capable of homomerisation. To the left: In vitro interaction between GST-tagged dFMR1 $1_{-\mathrm{VSA}}$ and dFMR1 ${ }_{+\mathrm{VSA}}$ produced in bacteria and their in vitro translated counterparts, human FMRP iso7 (hFMRP), and luciferase in presence of $150 \mathrm{mM} \mathrm{NaCl}$. In the Input lanes, $25 \%$ of the translation products used in a reaction were loaded. Amounts of dFMR1 proteins and human FMRP similar to those shown in the input lanes were retained by both GST-dFMR1s, indicating that approximately a quarter of in vitro translated material bound, respectively. Hence, homomerisation occurs independent of the dFMR1 splice variation. None of the proteins was unspecifically retained by GST alone. Luciferase, the negative control, was neither bound by $\mathrm{dFMR} 1_{-\mathrm{VSA}}$ nor $\mathrm{dFMR} 1_{+\mathrm{VSA}}$. To the right: A parallel experiment performed in presence of $200 \mathrm{mM}$ revealed that homomerization of dFMR1 ${ }_{-\mathrm{VSA}}$ and dFMR $1_{+\mathrm{VSA}}$ is sensitive to higher salt concentrations.

which reflects physiological conditions. More than $25 \%$ of in vitro translated dFMR1 proteins bound to their GST-tagged counterparts, independent of the splice variants used. Human FMRP displays similar binding efficiency to both variants of GST-dFMR1. The negative control was not retained by either GSTdFMR1 isoform and none of the three proteins was non-specifically bound by GST alone. In conclusion, dFMR1 protein is capable of homomerisation, like its human orthologues, and homomerisation ability is not influenced by the VSA splice variation.

Recently, Wan et al. (2000) did not to detect homomerization of $\mathrm{dFMR} 1_{+\mathrm{VSA}}$ in a similar experiment that has been carried out in higher salt conditions (200 $\mathrm{mM}$ ). Indeed, we also observed that homomerisation was sensitive to salt conditions above the physiological level (Fig. 2B, right panel).

\section{dFMR 1 Expression during Embryonic Development}

dFMR1 expression has been described to be unchanged throughout embryogenesis (Wan et al., 2000). However, in our hands, dFMR1 expression increases as development proceeds, as monitored by Western blot analysis (Fig. 2A). These data were confirmed by in situ hybridization on whole mount embryos (Figs. 


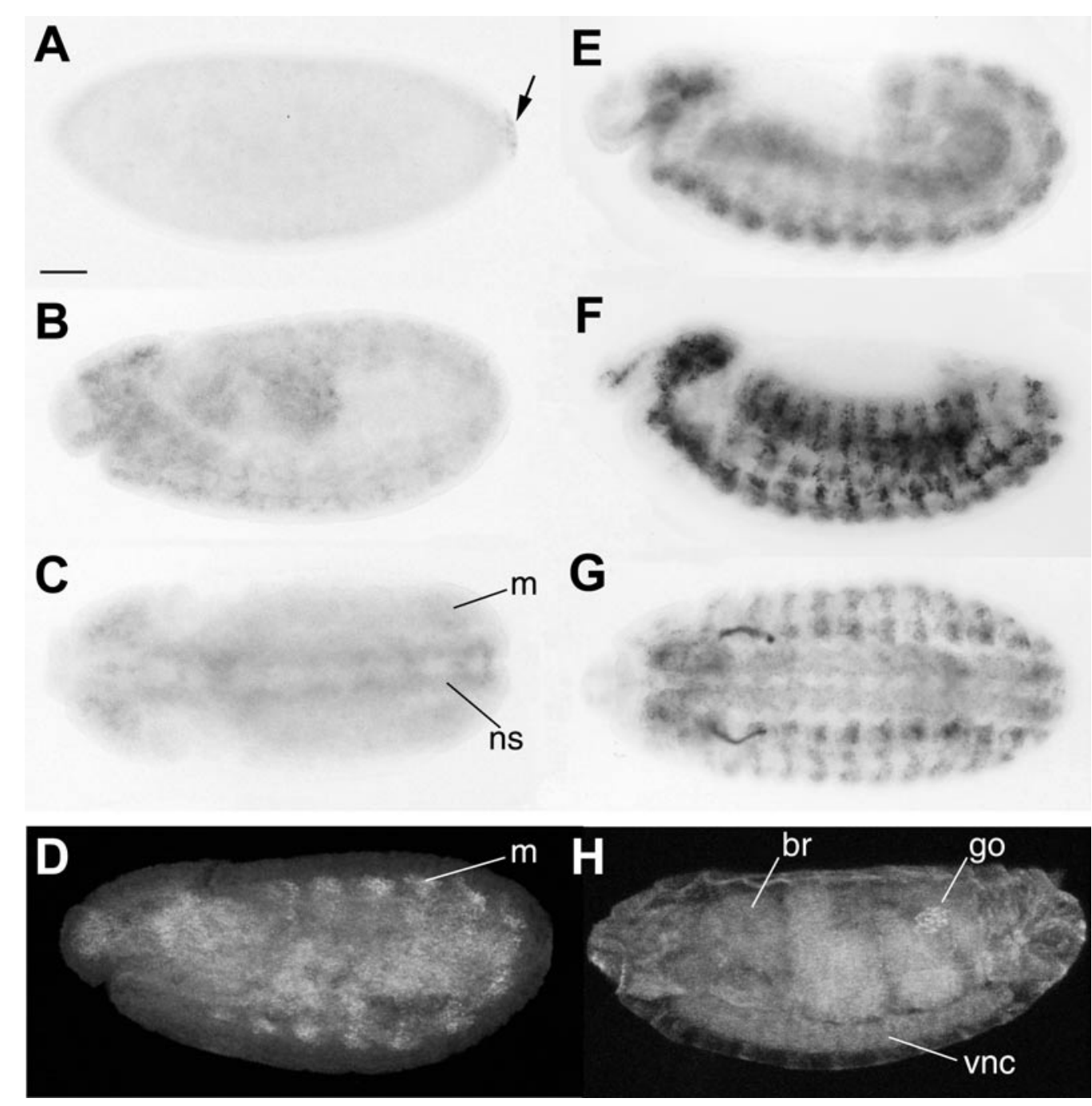

FIG. 3. dFMR1 expression during embryonic development. (A-C, E-G) In situ hybridisations using a dFMR1 specific probe and (D, H) immunohistochemistry using pAb \#1717. Anterior is to the left. (C, G) show ventral views, all others show lateral views. (A) Stage 5; dFMR1 transcript is present in the whole embryo and enriched at pole cells (see arrow). (B, C) Stage 10, dFMR1 mRNA is detected in the nervous system (ns) and at lower intensity in the somatic musculature (m). (D) Stage 11, the protein is predominantly detected in the latter. (E) Stage 12, dFMR1 mRNA labeling appears most strongly in the lateral stripes of the somatic musculature, in the nervous system (brain and ventral nerve cord), in the gut, and in the gonads. (F, G, H) Stage 16; mRNA and protein labeling. (br) brain, (vnc) ventral nerve cord, (go) gonads. Scale bar, $50 \mu \mathrm{m}$.

3A-3C, 3E-3G). Furthermore, since ms analysis confirmed the specificity of pAb 1717 , we used this antibody in immunohistochemistry (Figs. 3D and 3H). In the early embryo (stage 5), dFMR1 transcripts are present in the whole embryo at low levels, but abundant in pole cells (Fig. 3A, arrow), the germ line precursors. At stage 9, dFMR1 mRNA was detected in the nervous system and at lower level in the mesoderm (Figs. 3B and 3C). At this stage as well as later in embryogenesis, we detected the dFMR1 protein pre- dominantly in the somatic musculature (Fig. 3D). By stage 12, dFMR1 transcripts and protein begin to accumulate in the developing brain and ventral cord, as already described (Figs. 3E-3H (Wan et al., 2000)). From stage 13 on, increasing expression can also be observed in a pattern of lateral stripes, representing mostly somatic muscles (Figs. 3E-3G). Immunolabeling experiments using pAb 1717 (Fig. 3H) also showed that gonads, pharyngeal muscles, visceral muscles, and hind gut express dFMR1 at high levels. dFMR1 
signal at very early embryonic stages, detected by in in situ hybridization and Western blot analysis, suggests a maternal contribution of dFMR1.

At higher magnification and using confocal microscopy, a pattern of segmentally repeated structures at the lateral side of the embryo was labelled by $\mathrm{pAb}$ 1717 (Fig. 4A). To identify these structures, we performed colocalization studies with neuronal markers. Double labeling with mAb 22C10 detecting the microtubule-associated Futsch protein (Hummel et al., 2000) revealed that the six labeled dots (Fig. 4A, white double-arrows) correspond to the dendritic tips of the six abdominal neurons, belonging to proprioceptive chordotonal organs (Fig. 4D). dFMR1 also localises at the dendritic tips of the thoracic chordotonal organs (data not shown). In the first instar larvae, this signal appears expanded, labeling the entire dendrite of chordotonal neurons (data not shown). Double labeling with a nuclear neuronal marker (anti-Elav) also showed a close association of a dFMR1-positive longitudinal structure (Fig. 4A, large white arrow) with the nucleus of the lateral trachea-innervating dendrite (td) neuron (Fig. 4C). The dendrites of this neuron have been shown to innervate the trachea (Bodmer \& Jan, 1987), and indeed we found dFMR1 labeling at the same position.

We also detected dFMR1 in the somatic musculature, where it is enriched at the myotube extremity, which corresponds to the site of muscle attachment to the epidermis (Figs. 4A and 4B, yellow arrows and arrowheads). Finally, we found dFMR1 labelling in epidermal stripes at the segmental borders. These stripes most likely correspond to epidermal muscle attachment cells also called tendon cells (Volk, 1999) (Fig. 4A, blue arrow).

\section{dFMR 1 Expression in Larvae}

In the last stage of larval development, the third instar larvae, dFMR1 is predominantly present in the central nervous system, in the developing eye disc and in the testis (Fig. 5). Expression of dFMR1 in muscles persists in larvae, but is less prominent than during embryogenesis (not shown). In the brain, we observed strong labeling in the so-called mushroom body (Figs. $5 \mathrm{~A}$ and $5 \mathrm{~B})$, a structure that has been associated with some aspects of learning and memory in the adult (Zars, 2000). In Drosophila, each brain hemisphere contains the Kenyon cell bodies $(\mathrm{Kcb})$, which extend their dendrites and axons within the mushroom body. The dendrites make up a structure called calyx (ca), located just below the cell bodies, whereas the Kenyon cell axons build the $\alpha$ - and $\beta$-lobes of the larval mushroom body. dFMR1 accumulates within the Kenyon cell bodies, in its dendrites and axons. In addition, dFMR1 is present at moderate levels in a number of areas of the fly brain. In the ventral nerve cord, dFMR1 labeling is widespread and is localized in the cytoplasm (Fig. 5C). Two neurons (as revealed by Elav double labeling, data not shown) close to the midline show particular strong expression of dFMR1 (Fig. 5c, arrow).

High levels of dFMR1 protein were also observed in Drosophila testis, paralleling the human situation where the strongest FMRP expression is observed in brain and testes (Devys et al., 1993). In Drosophila, dFMR1 is strongly present the cytoplasm of spermatocytes, which make the middle three fifth of the testis, as shown in Fig. 5D (dFMR1 in red). We found practically no expression in spermatogonia (in the apical pole, to the left) nor in terminal cells (basal pole, to the right).

In the Drosophila eye disc, dFMR1 is expressed within the morphogenetic furrow (Fig. 5E, white arrow), where cells enter differentiation, as well as in the differentiated ommatidia. Double labeling with antiElav (green), which recognizes all photoreceptors, and anti-dFMR1 revealed a very apical, central, position of dFMR1, suggesting that dFMR1 is expressed in the R8 photoreceptor (Fig. 5F). Interestingly, we also observed moderate levels of dFMR1 in the Bolwig's nerve, where it is present along the entire axon of the larval photosensitive organ (Fig. 5E, arrowheads).

\section{DISCUSSION}

\section{Novel Features of an FXR Family Member}

In recent years, Drosophila melanogaster has been used successfully as a model for a variety of human neuronal pathologies (for a review see (Fortini \& Bonini, 2000)). In this study, we developed polyclonal antibodies against dFMR1, the Drosophila orthologue of the fragile X Mental Retardation protein. This allowed us to define the existence of two dFMR1 forms with apparent molecular weights of 85 and $92 \mathrm{kDa}$ and to analyze their developmental expression profile. Overexpression of a dFMR1 cDNA construct revealed that both bands can derive from the same coding sequence. Furthermore, using mass spectrometry analysis, we have shown that both $85-$ and $92-\mathrm{kDa}$ bands represent the dFMR1 full-length protein. Therefore we speculate that a posttranslational modification 

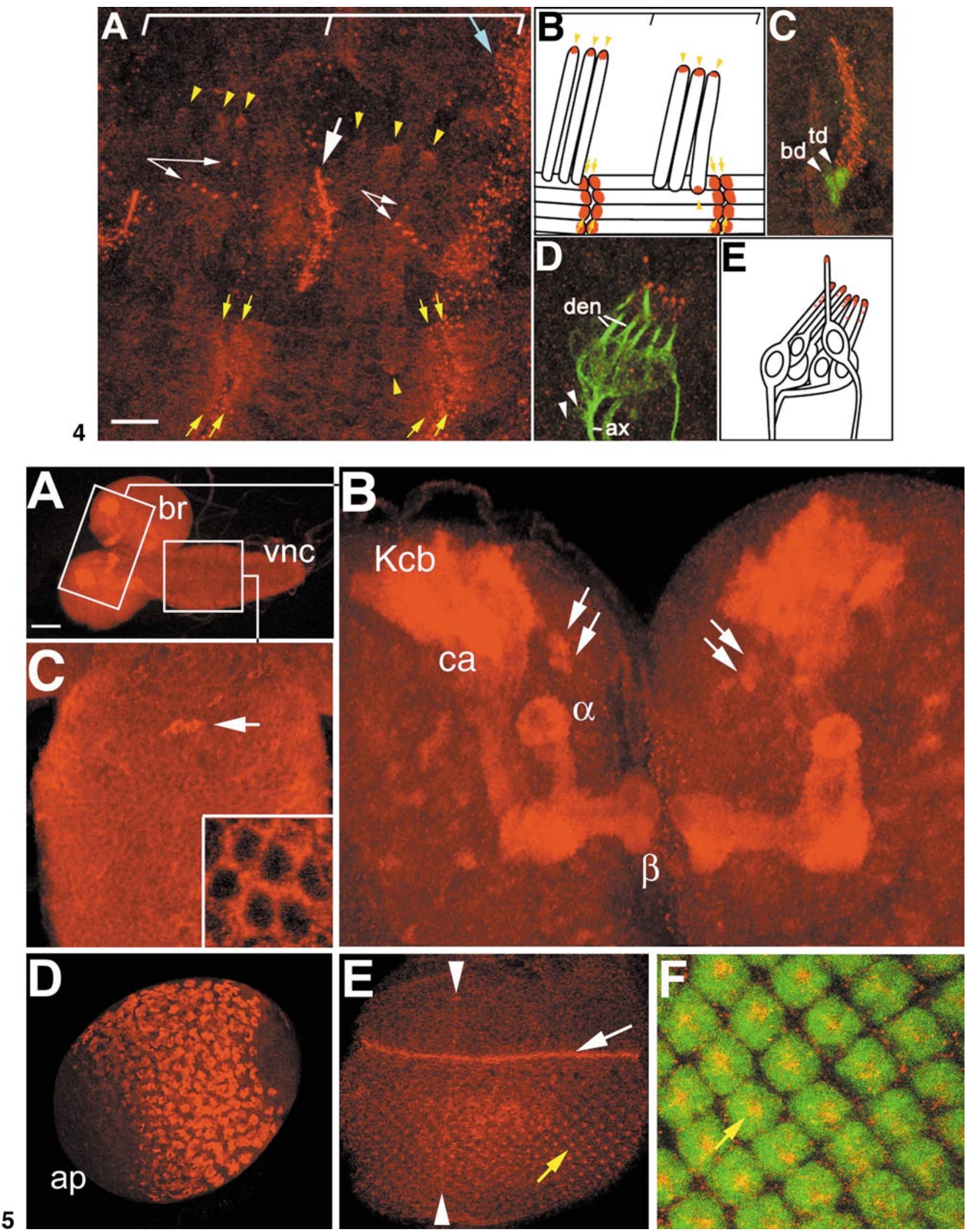
gives raise to the protein with the apparent molecular weight of $92 \mathrm{kDa}$. Several modifications like phosphorylation, acetylation, hydroxylation, isoprenylation have been reported to efficiently take place also in the reticulocyte lysate system (Ivan et al., 2001; Smutzer, 2001; Moraleda et al., 1999). This may explain why the $85 / 92-\mathrm{kDa}$ doublet has also been detected after in vitro translation. The dynamic regulation of the 92-kDa form during embryogenesis strongly suggests that it has a functional relevance and thus it will be important to determine the nature of the modification. In humans, the existence of multiple protein isoforms might have prevented the observation of such modifications.

RNA and protein analyses also revealed the existence of two different isoforms due to alternative splicing, resulting in presence or absence of three amino acids. These three aa are inserted at the end of the second $\beta$-fold of the $\mathrm{KH} 2$ domain, a region extremely conserved among the FXR protein family during evolution and in KH domains of other RNA-binding proteins (Musco et al., 1996). The same type of alternative splicing-alternative exon junction leading to insertion or omission of three amino acids-in the Wilm's tumor suppressor gene Wt1 has recently been demonstrated to modulate binding affinity to both, interacting proteins and nucleic acid, with profound consequences for subnuclear distribution and biological functions of the Wt1 protein (Hammes et al., 2001; Hastie, 2001). Thus, we argue that the three amino acid insertion in the $\mathrm{KH} 2$ domain of dFMR1 could also have important implications in its RNA-binding properties and function.

\section{Subcellular Distribution of dFMR 1}

We have analyzed in detail the embryonic and larval profile of expression by using polyclonal antidFMR1 antibody 1717. Labeling accumulates at the dendritic tips of chordotonal neurons and in a longitudinal structure associated with the lateral td neuron, a neuron whose two dendrites have been reported to innervate a trachea branch. Indeed, this structure is associated with the trachea sc branch (data not shown) and resembles mammalian dendrites spotted with dendritic spines, suggesting dFMR1 labeling of the td neuron dendrites. Labeling was also detected in many visceral and somatic muscles. In humans, FXR1P but not FMRP is expressed in skeletal muscle. These data suggest that dFMR1 recapitulates the localisation of all three of its human orthologues. It is worth noting that dFMR1 is not uniformously distributed in myotubes, but appears enriched at extremities, where muscles attach to epidermis. Other proteins, for example Kinesin and Nod, which also display polarized expression in neurons, have been shown to localise either at muscles attachment regions or in the interior of the muscle fibers (Clark et al., 1997). In the Drosophila larvae, we found strong labelling in spermatocytes. At this stage, spermatocytes have not yet entered meiosis, so that expression in mature spermatids could not be assessed. In humans, FMRP is present only in spermato-

FIG. 4. dFMR1 expression at the lateral side of stage 16 embryos. Anterior to the left, dorsal to the top. Anti-dFMR1 labeling is shown in red. Pictures represent projections of several confocal images. (A) two abdominal segments (see white bars at the top) showing the six spots (white double-arrows) and the longitudinal structure (large white arrow). The blue arrow points to epidermal labeling at the segmental border, which, by the position, corresponds to muscle attachment cells. In the more anterior segment, this labeling is not in focus. dFMR1 localises to the attachment sites of muscles (yellow arrows and arrowheads, see also B). (B) schematic representation of A with enriched dFMR1 labeling (in red) in the muscles attachment sites of the lateral transverse muscles (LT1-3, yellow arrowheads) and those of the ventral longitudinal muscles (VL1-4, yellow arrows). (C) Double labeling with anti-dFMR1 and anti-Elav (green), which specifically recognizes neuronal nuclei. (td) indicates the lateral trachea innervating neuron, $(\mathrm{bd})$ the the lateral bidendrite neuron. (D) Double labeling with anti-dFMR1 and the neuronal marker 22C10 (green). Note dFMR1 localisation at the dendritic tips of the abdominal chordotonal organs. (den) dendrites, (ax) axons. Arrowheads indicate the positions of the td and bd neurons shown in B. (E) schematic representation of the six chordotonal neurons shown in D. Scale bar, $10 \mu \mathrm{m}$.

FIG. 5. dFMR1 expression in larval tissues. Central nervous system, testis, and eye disc labeled with pAb \#1717 (red). (A) Low magnification of the brain (br)-ventral nerve cord (vnc) complex. White boxes indicate the regions presented in B and C at higher magnification. (B) dFMR1 pattern of expression in the brain. Kcb: Kenyon cell bodies, ca: calyx, $\alpha$ and $\beta$ : $\alpha$ - and $\beta$-lobes of the mushroom body. Arrows indicate two neurons with particular high dFMR1 expression in each brain hemisphere, located between $\alpha$-lobe and the Kenyon cell cluster. (C) Anterior part of the ventral nerve cord. Note the strong dFMR1 labeling in two neurons (arrow) close to the midline and the widespread cytoplasmic signal at moderate level (high magnification in the inset). (D) The apical pole (ap) of the testis containing spermatogonia is to the left. dFMR1 is expressed in spermatocytes. (E) DFMR1 in the eye disc: the protein is present at the morphogenic furrow (white arrow), along the Bolwig's nerve (white arrowheads) and in maturing ommatidia (yellow arrow, see also F). (F) High magnification of E at the level of 8-cell cluster ommatidia. Green color corresponds to the Elav protein, expressed in all photoreceptors. In contrast, dFMR1 (red) labeling appears restricted to one photoreceptor. Scale bar (in A): $95 \mu \mathrm{m}$ in A, $16 \mu \mathrm{m}$ in B, $24 \mu \mathrm{m}$ in C (2 $\mu \mathrm{m}$ in the inset), $40 \mu \mathrm{m}$ in D, $24 \mu \mathrm{m}$ in E, $5 \mu \mathrm{m}$ in F. 
gonia, but not at later stages (spermatocytes, spermatids), whereas expression of the FXR1P and FXR2P is shifted to later stages of maturation (Tamanini et al., 1997; Bakker et al., 2000). Thus, the expression pattern in testis resembles more that of FXR1P and FXR2P than that of FMRP.

The presence of strong labeling in the larval mushroom body is a very interesting finding. A variety of studies have implicated the adult mushroom body neuropile in several aspects of learning behaviour (for review see (Zars, 2000)). The structure of insect mushroom bodies, their development and the position of intrinsic neurons with its elaborations are well established (Heisenberg, 1998; Zars, 2000), which allowed us to show that anti-dFMR1 recognizes Kenyon cell bodies, as well as in their dendrites and axons. This data, together with 1717 labeling in the Bolwig's nerve, suggest that FMRP-like proteins can also localize to the axonal compartment. In the future, it will be interesting to examine whether axonal localisation is a feature specific to the Drosophila orthologue, or whether the human FXR proteins are also present in some axons.

Our expression study lays the basis for later analyses of dFMR1 mutants, generation of which is currently underway. To extend this Drosophila model, it will be important to determine whether orthologues of proteins already known to physically interact with FMRP (Bardoni et al., 1999; Schenck et al., 2001) have a conserved function in flies. Among the interacting proteins is CYFIP1 (Schenck et al., 2001), a protein that links FMRP to small GTPases known for their regulatory function in actin reorganisation, neuronal development and cognition (Luo, 2000). Drosophila genetics promise a fast dissection of the pathway comprising these proteins. Those experiments should shed light on the mechanisms responsible for abnormal spine development in the absence of the FMRP protein. Since synaptic defects have been observed in Fragile $X$ patients (Irwin et al., 2000), these studies will also contribute to our understanding of mechanisms underlying synaptic plasticity.

\section{ACKNOWLEDGMENTS}

We wish to thank J. L. Mandel for his support throughout the project. We are indebted to J. L. Mandel, M. Kammerer, E. Lalli, N. Roy, and unknown referees for helpful discussions and comments on the manuscript, and especially to J. M. Strub for MS analysis. We thank G. Duval, M. Boegler, D. Hentsch, and J. L. Vonesch for help with antibody production and confocal microscopy, and S. Benzer and G. Rubin for providing 22C10 and anti-Elav antibodies, respec- tively. This study was supported by funds from the Human Frontier Science Program (RGP0052/2001), Advisory Child Health and Human Development Council (R01 HD40612-01), institut National de la Santé et de la Recherche Médicale, the Centre National de la recherche Scientifique, and by the Hôpital Universitaire de Strasbourg (HUS). A.S. is supported by the Ernst Schering Research Foundation.

Note added in proof. Analysis of the second polyclonal antibody against dFMR1 (pAb \#1718) revealed ubiquitous protein distribution throughout the larval nervous system, the labelling being confined to the cell somata. Moreover, the recent availability of dFMR1 null flies (Zhang et al., Cell. 2001 107, 591-603) allowed us to show that pAb \#1717 mushroombody labelling is still present in the mutant (line 50M). Altogether, these data suggest that the \#1717 antibody recognizes dFMR1 as well as (an)other putative, dFMR1like, molecule(s).

\section{REFERENCES}

Ashley, C. T., Jr., Wilkinson, K. D., Reines, D., \& Warren S. T. (1993) FMR1 protein: Conserved RNP family domains and selective RNA binding. Science 262, 563-566.

Bakker, C. E., de Diego Otero, Y., Bontekoe, C., Raghoe, P., Luteijn, T., Hoogeveen, A. T., Oostra, B. A., \& Willemsen, R. (2000) Immunocytochemical and biochemical characterization of FMRP, FXR1P, and FXR2P in the mouse. Exp. Cell Res. 258, 162-170.

Bardoni, B., Mandel, J. L., \& Fisch, G. S. (2000) FMR1 gene and Fragile X Syndrome. Am. J. Med. Genet. (Semin. Med. Genet.) 97, 153-163.

Bardoni, B., Schenck, A., \& Mandel, J. L. (1999) A novel RNAbinding nuclear protein that interacts with the fragile $X$ mental retardation (FMR1) protein. Hum. Mol. Genet. 8, 2557-2566.

Bernardoni, R., Vivancos, B., \& Giangrande, A. (1997) glide/gcm is expressed and required in the scavenger cell lineage. Dev. Biol. 191, 118-130.

Bodmer, R., \& Jan, Y. N. (1987) Morphological differentiation of the embryonic peripheral neurons in Drosophila. Roux's Arch. Dev. Biol. 196, 69-77.

Brown, V., Jin, P., Ceman, S., Darnell, J. C., O’Donnell, W. T., Tenebaum, S. A., Jin, X., Wilkinson, K. D., Keene, J. D., \& Darnell, R. B. (2001) Microarray identification of FMRP-associated brain mRNAs and altered mRNA translational profiles in fragile $X$ syndrome. Cell 107, 12-20.

Clark, I. E., Jan, L. Y., \& Jan, Y. N. (1997) Reciprocal localization of Nod and kinesin fusion proteins indicates microtubule polarity in the Drosophila oocyte, epithelium, neuron and muscle. Development 124, 461-470.

Comery, T. A., Harris, J. B., Willems, P. J., Oostra, B. A., Irwin, S. A., Weiler, I. J., \& Greenough, W. T. (1997) Abnormal dendritic spines in fragile $\mathrm{X}$ knockout mice: Maturation and pruning deficits. Proc. Natl. Acad. Sci. USA 94, 5401-5404.

Darnell, J. C., Jensen, K. B., Jin, P., Brown, V., Warren, S. T., \& Darnell, R. B. (2001) Fragile X Mental retardation Protein targets G quartet mRNAs important for neuronal function. Cell 107, 1-11.

De Santa Barbara, P., Bonneaud, N., Boizet, B., Desclozeaux, M., Moniot, B., Sudbeck, P., Scherer, G., Poulat, F., \& Berta, P. (1998) Direct interaction of SRY-related protein SOX9 and steroidogenic factor 1 regulates transcription of the human anti-Mullerian hormone gene. Mol. Cell. Biol. 18, 6653-6665. 
Devys, D., Lutz, Y., Rouyer, N., Bellocq, J. P., \& Mandel, J. L. (1993) The FMR-1 protein is cytoplasmic, most abundant in neurons and appears normal in carriers of a fragile $\mathrm{X}$ premutation. Nature Genet. 4, 335-340.

Fortini, M. E., \& Bonini, N. M. (2000) Modeling human neurodegenerative diseases in Drosophila: on a wing and a prayer. Trends Genet. 16, 161-167.

Gadroy, P., Stridsberg, M., Capon, C., Michalski, J. C., Strub, J. M., van Dorselaer, A., Aunis, D., \& Metz-Boutigue, M. H. (1998) Phosphorylation and O-glycosylation sites of human chromogranin a from urine of patients with carcinoid tumors. J. Biol. Chem. 273, 34087-34097.

Greenough, W. T., Klintsova, A. Y., Irwin, S. A., Galvez, R., Bates, K. E., \& Weiler, I. J. (2001) Synaptic regulation of protein synthesis and the fragile X protein. Proc. Natl. Acad. Sci. USA 98, 7101-7106.

Hammes, A., Guo, J. K., Lutsch, G., Leheste, J. R., Landrock, D., Ziegler U., Gubler, M. C., \& Schedl, A. (2001) Two splice variants of the Wilms' tumor 1 gene have distinct functions during sex determination and nephron formation. Cell 106, 319-329.

Hastie, N. D. (2001) Life, sex, and wt1 isoforms—-three amino acids can make all the difference. Cell 106, 391-394.

Heisenberg, M. (1998) What do the mushroom bodies do for the insect brain? An introduction. Learn. Mem. 5, 1-10.

Hummel, T., Krukkert, K., Roos, J., Davis, G., \& Klambt, C. (2000) Drosophila Futsch/22C10 is a MAP1B-like protein required for dendritic and axonal development. Neuron 26, 357-370.

Irwin, S. A., Galvez, R., \& Greenough, W. T. (2000) Dendritic spine structural anomalies in fragile- $X$ mental retardation syndrome. Cerebral Cortex 10, 1038-1044.

Ivan, M., Kondo, K., Yang, H., Kim, W., Valiando, J., Ohh, M., Salic, A., Asara, J. M., Lane, W. S., \& Kaelin, W. G., Jr. (2001) HIFalpha targeted for VHL-mediated destruction by proline hydroxylation: Implications for $\mathrm{O} 2$ sensing. Science 292, 464-468.

Laggerbauer, B., Ostareck, D., Keidel, E.-M., Ostareck-Lederer, A., \& Fischer, U. (2001) Evidence that fragile X mental retardation protein is a negative regulator of translation. Hum. Mol. Genet. 10, 329-338.

Li, Z., Zhang, Y., Ku, L., Wilkinson, K. D., Warren, S. T., \& Feng, Y. (2001) The fragile $X$ mental retardation protein inhibits translation via interacting with mRNA. Nucleic Acids Res. 29, 2276-2283.

Luo, L. (2000) Rho GTPases in neuronal morphogenesis. Nat. Rev. Neurosci. 1, 173-180.

Moraleda, G., Seeholzer, S., Bichko, V., Dunbrack, R., Otto, J., \& Taylor, J. (1999) Unique properties of the large antigen of hepatitis delta virus. J. Virol. 73, 7147-7152.

Musco, G., Stier, S., Joseph, C., Castiglione Morelli, M. A., Nilges, M., Gibson, T. J., \& Pastore, A. (1996) Three-dimensional structure and stability of the $\mathrm{KH}$ domain: Molecular insights into the fragile X syndrome. Cell 85, 237-245.

Schaeffer, C., Bardoni, B., Mandel, J. L., Ehresmann, B., Ehresmann, C., \& Moine, H. (2001) The Fragile X mental retardation protein interacts specifically with its own mRNA via a purine-quartet structure. EMBO J. 20, 4803-4813.

Schenck, A., Bardoni, B., Moro, A., Bagni, C., \& Mandel, J. L. (2001) A highly conserved protein family interacting with the fragile $X$ Mental Retardation Protein and displaying selective Interactions with the FMRP related proteins FXR1P and FXR2P. Proc. Natl. Acad. Sci. USA 98, 8844-8849.

Siomi, H., Siomi, M. C., Nussbaum, R. L., \& Dreyfuss, G. (1993) The protein product of the fragile $\mathrm{X}$ gene, FMR1, has characteristics of an RNA-binding protein. Cell 74, 291-298.

Siomi, M. C., Zhang, Y., Siomi, H., \& Dreyfuss, G. (1996) Specific sequences in the fragile $X$ syndrome protein FMR1 and FXR proteins mediate their binding to $60 \mathrm{~S}$ ribosomal subunits and the interactions among them. Mol. Cell. Biol. 16, 3825-3832.

Smutzer, G. (2001) Cell-free Transcription and Translation. Scientist $15,22$.

Sung, Y.-J., Conti, J., Currie, J. R., \& Denman, R. B. (2000) RNAs that interact with the fragile-X syndrome RNA binding protein FMRP. Biochem. Biophys. Res. Commun. 275, 973-980.

Tamanini, F., Willemsen, R., van Unen, L., Bontekoe, C., Galjaard, H., Oostra, B. A., \& Hoogeveen, A. T. (1997) Differential expression of FMR1, FXR1 and FXR2 proteins in human brain and testis. Hum. Mol. Genet. 6, 1315-1322.

Vincent, S., Vonesch, J. L., \& Giangrande, A. (1996) Glide directs glial fate commitment and cell fate switch between neurones and glia. Development 122, 131-139.

Volk, T. (1999) Singling out Drosophila tendon cells: A dialogue between two distinct cell types. Trends Genet. 15, 448-453.

Wan, L., Dockendorff, T. C., Jongens, T. A., \& Dreyfuss, G. (2000) Characterization of dFMR1, a Drosophila Melanogaster homolog of the fragile X mental retardation protein. Mol. Cell. Biol. 20, 8536-8547.

Weiler, I. J., Irwin, S. A., Klintsova, A. Y., Spencer, C. M., Brazelton, A. D., Miyashiro, K., Comery, T. A., Patel, B., Eberwine, J., \& Greenough, W. T. (1997) Fragile X mental retardation protein is translated near synapses in response to neurotransmitter activation. Proc. Natl. Acad. Sci. USA 94, 5395-5400.

Zars, T. (2000) Behavioral functions of the insect mushroom bodies. Curr. Opin. Neurobiol. 10, 790-795.

Zhang, Y., O'Connor, J. P., Siomi, M. C., Srinivasan, S., Dutra, A., Nussbaum, R. L., \& Dreyfuss, R. (1995) The fragile X mental retardation syndrome protein interacts with novel homologs FXR1 and FXR2. EMBO J. 14, 5358-5366. 